\title{
Joint Optimization of Rate Allocation and BLAST Ordering to Minimize Outage Probability
}

\author{
Arumugam Kannan, Badri Varadarajan and John R. Barry \\ School of Electrical and Computer Engineering \\ Georgia Institute of Technology, Atlanta, GA 30332-0250 USA \\ \{aru, badri, barry\}@ece.gatech.edu
}

\begin{abstract}
We consider a wireless communication system over a multiple-input multiple-output Rayleigh-fading channel with successive-cancellation detection. The outage probability of such a system is strongly dependent on two choices: the order in which the layers are detected, and the rate-allocation strategy at the transmitter. We propose the rate-normalized ordering algorithm, a generalization of the BLAST ordering algorithm that is shown to minimize outage probability. We further optimize the allocation of rate and energy at the transmitter, for a variety of receiver ordering strategies. Finally, we jointly optimize the receiver ordering and transmitter rate and energy allocations. Our main conclusion is that, for a wide range of data rates and SNR, the outage probability is minimized by a combination of rate-normalized ordering and a partially uniform rate and energy (PURE) allocation strategy. The jointly optimum system outperforms the Bell Labs layered space-time (BLAST) architecture by $15 \mathrm{~dB}$ at $8 \mathrm{~b} / \mathrm{s} / \mathrm{Hz}$ and an outage probability of $10^{-3}$, when operating over a 4-input 4-output Rayleigh-fading channel. Also, the jointly optimum system shows an improvement of $1.5 \mathrm{~dB}$ over a recently proposed combination of optimum allocation and fixed ordering.
\end{abstract}

\section{INTRODUCTION}

The use of multiple transmit and receive antennas in wireless communication systems offers dramatic multiplexing and diversity gains, enabling high spectral efficiencies and low error rates over wireless fading channels. The transmitter and receiver should be appropriately designed to exploit the gains offered by multiple-input, multiple-output (MIMO) systems. Further, system design should aim for low computational complexity.

The BLAST architecture [1][2] achieves a high multiplexing gain by transmitting independent data streams, possibly coded, from each transmit antenna. The BLAST receiver employs the ordered successive cancellation (SC) decoding algorithm, which maximizes the minimum SNR among all stages of decoding. The $\mathrm{SC}$ receiver is much less complex than the optimum joint ML decoder, but it does not fully exploit the diversity gain offered by the MIMO channel. Consequently, the BLAST system suffers from high error rates, even at high SNR.

This research was supported in part by National Science Foundation grants CCR-0082329 and CCR-0121565.
The error performance of the SC decoder can be improved by rate and energy allocation across the various antennas. Ideally, the optimum allocation is obtained through a feedback path from receiver to transmitter, where either the channel itself or the optimum allocation, computed by the receiver, is fed back [3][4][5]. Transmitter allocation strategies based on receiver feedback are effective, but such feedback is not always available in practical systems.

Instead of relying on feedback, one can allocate rates and energies based on the statistics [6][7] of the random channel, which may be known to the transmitter even if the instantaneous channel is not. The loading strategy that minimizes the error probability, for the fixed case where the receiver performs no ordering, was obtained in [6]. The outage capacity of such a system was analyzed in [8]. A strategy which selects a subset of the transmit antennas based on channel statistics was shown [9][10] considerably improve the performance of the conventional BLAST system. However, the jointly optimal transmitter receiver structure has not yet been explored.

In this work, we aim to minimize outage probability. The outage probability is a lower bound on the word error rate achievable by a coded MIMO system. The bound is tight when the outer error-correcting outer code is powerful, in the sense that it approaches capacity over an AWGN channel.

In this paper, we propose the rate-normalized ordering algorithm, which minimizes the outage probability for any given transmitter loading strategy. Second, we obtain the transmitter rate and energy allocation that minimizes the outage probability with rate-normalized ordering. Our main conclusion is that, for a wide range of data rates and SNR, the outage probability is minimized by a partially uniform rate and energy (PURE) allocation strategy, which distributes the available rate and energy uniformly over a fraction of the available transmit antennas.

This paper is organized as follows. In Section II, we describe the channel model, and the system architecture. In Section III, we review the BLAST ordering algorithm, propose our new rate-normalized variation and prove its optimality. In Section IV, we discuss the problem of optimum rate allocation for each receiver ordering algorithm. The analysis is supported by simulation results in Section V. Finally, Section VI summarizes our conclusions. 


\section{System MOdel AND BACKGROUND}

We consider a MIMO system with $t$ transmit and $r$ receive antennas, with the assumption that $r \geq t$. In keeping with the BLAST architecture [1], independent data streams are transmitted on each transmit antenna. Before transmission, each data stream is encoded by an error-correcting code that is designed to approach capacity on an AWGN channel. The $i$-th data stream carries an information rate of $R_{i} \mathrm{~b} / \mathrm{s} / \mathrm{Hz}$ with an average energy of $E_{i}$. The total data rate is $R=\sum_{i=1}^{t} R_{i}$, and the average transmit energy is $E=\sum_{i=1}^{t} E_{i}$.

We assume that the channel is linear, flat-fading and quasistatic, so that the received vector at the $k$-th signaling interval is:

$$
\boldsymbol{y}_{k}=\mathbf{H} \boldsymbol{x}_{k}+\boldsymbol{n}_{k}
$$

The elements of the $r \times 1$ noise vector $\boldsymbol{n}_{k}$ are independent, circularly symmetric Gaussian random variables with zero mean and variance $N_{\mathrm{O}}$ so that $\mathrm{E}\left[\mathbf{n}_{k} \mathbf{n}_{l}{ }^{*}\right]=\delta_{k-l} N_{\mathrm{O}} \mathbf{I}_{r}$, where $\mathbf{A}^{*}$ denotes conjugate transpose of $\mathbf{A}$. The $r \times t$ channel matrix $\mathbf{H}$ is assumed to be a random Rayleigh fading matrix, its entries being independent, circularly symmetric complex Gaussian random variables with zero mean and unit variance. The receiver knows $\mathbf{H}$, but the transmitter has no information about $\mathbf{H}$. Under these assumptions, the SNR per receive antenna is given by $S=E / N_{\mathrm{O}}$.

In this paper, we focus on receivers which employ the successive cancellation (SC) decoding algorithm [1]. SC decoders decode one data stream at a time, subtracting out the estimated contribution of previously decoded data streams, and nulling out interference from undecoded streams.

An important degree of freedom in the design of SC decoders is the choice of the order in which the streams are decoded. Let $i_{j}$ denote the index of the symbol decoded in the $j$-th stage. To decode all the symbols $\left\{\hat{x}_{k}^{\left(i_{j}\right)}\right\}$ in the $i_{j}$-th data stream, the SC decoder cancels off the estimated contribution from the previously detected data streams to obtain

$$
\boldsymbol{r}_{k}^{(j)}=\boldsymbol{y}_{k}-\sum_{l=1}^{j-1} \boldsymbol{h}_{i_{l}} \hat{x}_{k}^{\left(i_{l}\right)}
$$

where $\boldsymbol{h}_{i}$ denotes the $i$-th column of the channel matrix $\mathbf{H}$. If previous decisions are correct, then $\left\{\boldsymbol{r}_{k}{ }^{(j)}\right\}$ contains contributions only from the stream of interest $i_{j}$, and interference from the undecoded streams. To null out the interference, the SC decoder uses the nulling vector $\boldsymbol{w}_{j}$, defined as the first row of the Moore-Penrose inverse of the $\operatorname{matrix}\left[\boldsymbol{h}_{i_{j}}, \boldsymbol{h}_{i_{j}+1}, \ldots, \boldsymbol{h}_{i_{t}}\right][1]$.

Using the nulling vector, the SC decoder obtains the $j$-th decision stream, $d_{k}^{(j)}=\boldsymbol{w}_{j}^{*} \boldsymbol{r}_{k}^{(j)}$. Assuming perfect decision feedback, the channel model reduces to

$$
d_{k}^{(j)}=x_{k}^{\left(i_{j}\right)}+\boldsymbol{w}_{j}^{*} \boldsymbol{n}_{k}
$$

The equivalent channel (3) is an AWGN channel with noise variance $N_{\mathrm{O}}\left\|\boldsymbol{w}_{j}\right\|^{2}$. The estimates $\left\{\hat{x}_{k}^{\left(i_{j}\right)}\right\}$ of the $i_{j}^{\text {th }}$ data stream are obtained from $\left\{d_{k}^{(j)}\right\}$.

We now proceed to quantify the error probability of the SC decoder, as a function of $\left\{R_{i}\right\},\left\{E_{i}\right\}$ and the decision ordering vector $\boldsymbol{i}=\left[i_{1}, i_{2}, \ldots, i_{t}\right]^{T}$. For convenience, define the inverse ordering vector $\boldsymbol{j}=\left[j_{1}, j_{2}, \ldots, j_{t}\right]^{T}$ such that $q=j_{i_{q}}$ for $q=1,2$, $\ldots, t$. The effective channel corresponding to the $i$-th symbol has a noise variance equal to $N_{\mathrm{o}} / \gamma_{i}$, where $\gamma_{i}$ is the SNR scaling factor given by $\gamma_{i}=1 /\left\|\boldsymbol{w}_{j_{i}}\right\|^{2}$. Recall that the $i$-th data stream has an average energy $E_{i}$, hence the instantaneous SNR of the effective channel is $E_{i} \gamma_{i} / N_{\mathrm{O}}$, and the instantaneous capacity is $\log _{2}\left(1+E_{i} \gamma_{i} / N_{\mathrm{O}}\right)$. Since each data stream is assumed to have a capacity-achieving code, it is incorrectly decoded if and only if an outage occurs, i.e., if and only if:

$$
\log _{2}\left(1+E_{i} \gamma_{i} / N_{\mathrm{O}}\right)<R_{i}
$$

or equivalently if and only if $\gamma_{i}$ is less than $1 / S N R_{i}^{\text {norm }}$, where $S N R_{i}^{\text {norm }}$ is the rate-normalized $S N R$ of the $i$-th data stream, as defined by Forney [11]:

$$
S N R_{i}^{\text {norm }}=\frac{E_{i} / N_{0}}{2^{R_{i}}-1} .
$$

For notational convenience, we define $\rho_{i}=S N R_{i}^{\text {norm }} \cdot$ The rate-normalized SNR characterizes the error performance of the system better than just the SNR, since it captures the effect of the data rate.

The overall SC decoder is a bank of parallel scalar decoders for each stream. If all data streams are outage-free, the SC decoder is also error-free. However, if any of the streams is in outage, the SC decoder is in outage, and hence has a non-zero probability of frame error. Consequently, the frame-error rate of the coded system is upper-bounded by the outage probability

$$
P_{\mathrm{o}}\left(\boldsymbol{i},\left\{\rho_{i}\right\}\right)=\operatorname{Pr}\left[\bigcup_{i=1}^{t}\left\{\gamma_{j_{i}}<\frac{1}{\rho_{i}}\right\}\right] \text {. }
$$

In the following sections, we will discuss problem of minimizing the outage probability by the optimal choice of $\left\{R_{i}\right\},\left\{E_{i}\right\}$ and the ordering vector $\boldsymbol{i}$.

\section{Receiver Design: Choice of Ordering Algorithm}

For every instance of the channel $\mathbf{H}$, the receiver uses an ordering algorithm to compute $\boldsymbol{i}$, which in turn determines the SNR scaling factors $\left\{\gamma_{i}\right\}$. Averaging over $\mathbf{H}$, the ordering algorithm determines the probability distribution of the SNR scaling vector $\Gamma=\left[\gamma_{1}, \gamma_{2}, \ldots \gamma_{t}\right]^{T}$, and hence the outage probability. In this section, we discuss three possible ordering algorithms and derive the outage probability for each. 


\section{III-A. Fixed Ordering}

The simplest ordering algorithm is fixed ordering, where the streams are decoded simply in the increasing order of their index, i.e., $\boldsymbol{i}=\boldsymbol{j}=[1,2, \ldots, t]^{T}$, irrespective of $\mathbf{H}$. In this case, it is well known [12] that the SNR scaling factors $\left\{\gamma_{i}\right\}$ are independent. Thus, the outage probability (6) reduces to

$$
P_{\text {fixed }}\left(\left\{\rho_{i}\right\}\right)=1-\prod_{i=1}^{t} \operatorname{Pr}\left[\gamma_{i} \geq \frac{1}{\rho_{i}}\right] .
$$

Further, from [12], $\gamma_{i}$ has a $\chi^{2}$-distribution with $2(r-t+i)$ degrees of freedom, hence

$$
\operatorname{Pr}\left[\gamma_{i} \geq \rho_{i}\right]=\exp \left(\frac{-1}{\rho_{i}}\right) \sum_{l=0}^{r-t+i} \frac{1}{l !}\left(\frac{1}{\rho_{i}}\right)^{l} .
$$

Substituting (8) in (7) gives a closed form expression for the outage probability of fixed ordering.

\section{III-B. BLAST Ordering}

The BLAST ordering algorithm [1] can be summarized as follows. Given $\mathbf{H}$, the first stream to be decoded, $i_{1}$, is chosen as the one with the nulling vector of least magnitude, i.e., the maximum post-nulling SNR. The next stream, $i_{2}$, is chosen to maximize $\gamma_{2}$, among the remaining $t-1$ choices, and so on. It was shown in [1] that this greedy ordering algorithm is also globally optimum, as stated below.

Remark 1. For stages $j=1,2 \ldots t$, the BLAST ordering algorithm chooses $i_{j}$ so as to achieve the maximum value of $\gamma_{j}$ among the $t-j+1$ possibilities. In the process, it also maximizes the minimum of SNR scaling factors, namely $\min \left(\gamma_{1}, \gamma_{2}, \ldots, \gamma_{t}\right)$.

The SNR scaling factors $\gamma_{1}, \gamma_{2}, \ldots, \gamma_{t}$ produced by BLAST ordering are not mutually independent. Therefore, obtaining a closed form expression for the density function of $\Gamma$ is an open problem. However, the following properties hold for the ordering vector $\boldsymbol{i}$ and the SNR scaling vector $\Gamma$.

Theorem 1. For a Rayleigh fading channel, the ordering vector $\boldsymbol{i}$ and the SNR scaling vector $\Gamma$ produced by the BLAST ordering algorithm are independent. Further, $\boldsymbol{i}$ is uniformly distributed over the set of all permutations of $[1,2, \ldots, t]^{T}$.

In Section IV-B, we will use the above facts to derive a simple expression for the union bound on the outage probability (6).

\section{III-C. Rate-Normalized Ordering}

In this section, we propose the rate-normalized (RN) ordering algorithm which minimizes the outage probability for any given transmitter rate allocation. First, note from (6) that an outage occurs if and only if $\min \left(\gamma_{j} \rho_{i_{j}}\right)$ across all the decoding stages is less than unity. From this observation, we state the following lemma.
Lemma 3.1. To minimize the outage probability (6), the ordering vector $\boldsymbol{i}$ should be chosen to ensure that $\min \left(\gamma_{j} \rho_{i_{j}}\right)$ is maximized over $j=1,2, \ldots, t$.

Theorem 2. At every stage of decoding $j=1,2, \ldots, t$, the rate-normalized ordering algorithm chooses $i_{j}$ so as to achieve the maximum value of $\gamma_{j} \rho_{i}$ among the $t-j+1$ possibilities. In the process, it maximizes the minimum of $\gamma_{j} \rho_{i_{j}}$ across all data streams.

Proof: Let the scaled channel matrix be $\mathbf{H}^{\prime}=\mathbf{H D}$, where $\mathbf{D}$ is a $t \times t$ diagonal matrix, whose $j$-th diagonal entry is $d_{j j}=\sqrt{\rho_{i_{j}}}$. If the QR decomposition of $\mathbf{H}$ is given by $\mathbf{H}=\mathbf{Q R}$, then the scaled channel matrix can be written as $\mathbf{H}^{\prime}=\mathbf{Q R D}=\mathbf{Q} \mathbf{R}^{\prime}$. It is well known [13] that the SNR scaling factors resulting from SC decoding of $\mathbf{H}$ are equal to the squared diagonal entries of $\mathbf{R}$. Therefore, the SNR scaling factor for the scaled channel $\mathbf{H}^{\prime}$ is simply $\gamma_{j}^{\prime}=$ $R_{j j}{ }^{2} d_{j j}{ }^{2}=\gamma_{j} \rho_{i_{j}}$. Hence, the minimum of $\gamma_{j}^{\prime}$ is maximized by employing the usual BLAST ordering algorithm of [1] on $\mathbf{H}^{\prime}$ instead of $\mathbf{H}$.

Combining Lemma 3.1 and Theorem 2, we conclude that the rate-normalized ordering algorithm minimizes the outage probability among all possible ordering algorithms.

\section{TRAnsmitter Design: \\ Optimal Rate and Energy Allocation}

In this section, we discuss the transmitter optimization problem, namely to choose the $\left\{R_{i}\right\}$ and $\left\{E_{i}\right\}$ to minimize the outage probability at a given SNR under the constraints that $\sum_{i=1}^{t} R_{i}=R$ and $\sum_{i=1}^{t} E_{i}=E$. We begin by stating the following remark about the optimum energy allocation holds for all ordering algorithms.

Remark 2. Suppose $\left\{R_{i}^{*}\right\}$ and $\left\{E_{i}^{*}\right\}$ are the rate and energy allocations that minimize the outage probability (6). They are related by:

$$
E_{i}^{*} / E=\frac{1-2^{-R_{i}^{*}}}{\sum_{j=1}^{t} 1-2^{-R_{j}^{*}}} .
$$

This is easily proved using Lagrange multipliers. The proof is omitted due to space constraints. The implication is that the transmitter optimization is simplified to one of choosing only the data rates $\left\{R_{i}\right\}$, with the optimum energies $E_{i}$ automatically determined by (9). Thus, the number of variables to be optimized is reduced from $2 t$ to $t$.

\section{$I V-A$. Fixed Ordering}

Transmitter optimization for fixed ordering has already been solved [6] except that the relation (9) between the optimum energy and rate allocations was not used. The optimum allocation is determined by a random search followed by constrained gradient descent over the space of all possible data rates $\left\{R_{i}\right\}$ that sum up to $R$. For example, 
consider a 4-input, 4-output MIMO system operating at a data rate of $R=8 \mathrm{~b} / \mathrm{s} / \mathrm{Hz}$ and an $\mathrm{SNR}$ of $S=15 \mathrm{~dB}$. Numerical optimization yields the optimum data rate allocation to be $\left\{R_{i}\right\}=\{0,1.31,2.99,3.70\}$. From (9), the corresponding energy allocation is $\left\{E_{i} / E\right\}=\{0,0.25,0.36,0.39\}$. Note that the streams detected later carry a higher data rate than streams detected early. This result is intuitively satisfying because, from (7) and (8), the diversity order of the $j$-th detected stream is $r-t+j$. It is intuitively pleasing that a higher fraction of the available bits are loaded into streams with higher diversity orders.

\section{IV-B. BLAST Ordering}

As stated in Section III-B, the SNR scaling factors produced by the BLAST ordering algorithm are dependent, and no closed-form expression is known for their probability distribution and hence the outage probability. Instead, we suggest using the union bound in (6) to perform transmitter optimization. From (6), it is clear that the outage probability is bounded by

$$
P_{\mathrm{UB}}\left(\left\{\rho_{i}\right\}, \boldsymbol{j}\right)=\sum_{i=1}^{t} \operatorname{Pr}\left[\gamma_{j_{i}}<\frac{1}{\rho_{i}}\right] .
$$

Each term in the summation can be split into an average over the stage $j_{i}$ in which the $i$-th stream is decoded, giving

$$
\operatorname{Pr}\left[\gamma_{j_{i}}<\frac{1}{\rho_{i}}\right]=\sum_{k=1}^{t} \operatorname{Pr}\left(j_{i}=k\right) \operatorname{Pr}\left[\gamma_{k}<\frac{1}{\rho_{i}} \mid j_{i}=k\right] .
$$

Theorem 1 states that $j_{i}$ is uniformly distributed over $\{1, \ldots t\}$, hence $\operatorname{Pr}\left(j_{i}=k\right)=1 / t$. Also from Theorem 1 , the SNR scaling factor is independent of $j_{i}$, thus conditioning on $j_{i}$ does not change the distribution of $\gamma_{k}$. Thus, (11) simplifies to

$$
\operatorname{Pr}\left[\gamma_{j_{i}}<\frac{1}{\rho_{i}}\right]=\frac{1}{t} \sum_{k=1}^{t} F_{k}\left(\frac{1}{\rho_{i}}\right)
$$

where we define the left-continuous cumulative distribution functions by $F_{k}(x)=\operatorname{Pr}\left[\gamma_{k}<x\right]$ for $k=1,2, \ldots, t$. Let $F(x)$ denote the average of these distribution functions over the $t$ symbols. From (12), $\operatorname{Pr}\left[\gamma_{j_{i}}<1 / \rho_{i}\right]=F\left(1 / \rho_{i}\right)$. Substituting in (10), we get the union bound on the outage probability to be

$$
P_{\text {Ub-BLAST }}\left(\left\{\rho_{i}\right\}\right)=\sum_{i=1}^{t} F\left(\frac{1}{\rho_{i}}\right) .
$$

An analytical expression for $F(x)$ is not known in closed form, so even the simplified union bound (13) cannot be evaluated as is. However, the function $F(x)$ can be numerically estimated as follows. A large number of Rayleigh fading matrices are generated, the BLAST algorithm is run for each one, and the resulting $\left\{\gamma_{i}\right\}$ are rounded off to preselected bins. Averaging this discrete approximation of the distribution function of $\left\{\gamma_{i}\right\}$, we get a discrete approximation to $F(x)$.
Note that (13) is just the sum of the same function evaluated for each of the terms $\left\{1 / \rho_{i}\right\}$. This implies that BLAST ordering treats all the data streams identically i.e., if the data rates and energies of two streams $i$ and $i^{\prime}$ are equal, then they make the same contribution $F\left(1 / \rho_{i}\right)$ to the union bound (13). From this observation, it is tempting to conclude that the minimizing solution is to allocate identical data rates and energies, $R / t$ and $E / t$ respectively, to all the streams. However, this conclusion is not valid because the function $F(x)$ is not necessarily convex.

For example, consider a 4-input, 4-output MIMO system operating at $8 \mathrm{~b} / \mathrm{s} / \mathrm{Hz}$ at an SNR of $20 \mathrm{~dB}$. For this system, we numerically estimated $F(x)$ and performed a random search for the optimum rate allocation. The uniform allocation yielded a union bound (13) equal to $3.4334 \times 10^{-2}$. However, the optimum allocation was found to be the partially uniform rate allocation $\{0,0,4,4\}$, which distributes the rate uniformly over two of the four transmit antennas. This allocation yielded a union bound of $1.1110 \times 10^{-3}$, which is significantly lower. Based on numerical experiments, we make the following conjecture about the optimal allocation.

Conjecture 1. The union bound for the BLAST-ordered SC decoder is minimized by a partially uniform rate and energy (PURE) allocation, with $K$ streams carrying a data rate of $R / K$ and energy of $E / K$, and the remaining $t-K$ data streams carrying zero data rate and zero energy.

Hence, numerical optimization reduces to finding the optimal number of active streams $K \in\{1,2, \ldots, t\}$. The optimum value is typically less than $t$ at high SNR, and it decreases with increasing SNR. This can be explained intuitively by the fact the diversity order is the key determinant of error performance at high SNR, and reducing the number of active inputs implies that the diversity gain of the SC decoded system is enhanced.

\section{IV-C. Rate-Normalized Ordering}

We now aim to find the optimum rate and energy allocation at the transmitter when the receiver employs the $\mathrm{RN}$ ordering algorithm of Section III-C.

The analytical expression for outage probability with RN ordering is intractable. Even the union bound is intractable, because the distributions of $\Gamma$ and $\boldsymbol{j}$ for the $\mathrm{RN}$ ordering algorithm depend on the rate allocations. However, based on heuristic observations, we make the following conjecture regarding the optimum data rate allocation.

Conjecture 2. The optimum data rate allocation for the rate-normalized ordering algorithm is either

- the optimum allocation for the case of fixed ordering, or

- a partially uniform rate and energy (PURE) allocation, where $K$ inputs carry a rate of $R / K$ each, and the rest carry zero data rate. 
According to Conjecture 2, one can restrict the search for the optimum data rate allocation to $t+1$ possibilities. Of particular interest in Conjecture 2 is the fact that a partially uniform allocation is often the optimum solution for $\mathrm{RN}$ ordering. It was discussed in Section IV-B that a PURE allocation is optimum for BLAST ordering. Note that, with a PURE allocation, RN ordering amounts exactly to BLAST ordering and for this reason, the PURE allocation is expected to be a good solution for RN ordering.

\section{NumericAl Results}

In this section we present numerical results for a $4 \times 4$ MIMO system operating at $R=8 \mathrm{~b} / \mathrm{s} / \mathrm{Hz}$, assuming independent Rayleigh fading.

We first consider a fixed ordering [6], and quantify the benefits of optimizing the rate and energy allocations. At $S=20 \mathrm{~dB}$ with a fixed ordering, the optimal rates and energies are $\{0,0,3.63,4.37\}$, and $\{0,0,0.49 E, 0.51 E\}$, respectively, which leads to an outage probability of 0.002422. In comparison, a uniform rate allocation with fixed ordering gives an outage probability of 0.1201 , about fifty times larger.

Fig. 1 compares the error performance of three ordering strategies at $R=8 \mathrm{~b} / \mathrm{s} / \mathrm{Hz}$ : the fixed ordering, the conventional BLAST ordering, and the rate-normalized ordering. All three systems use the same rate allocation $\left\{R_{i}\right\}=\{0,1.31,2.99,3.70\}$, which is a good candidate for comparison since it minimizes the outage probability of the fixed-ordering decoder at $S=15 \mathrm{~dB}$. It is seen from Fig. 1 that the rate-normalized ordering outperforms the optimized fixed ordered system by $1.5 \mathrm{~dB}$ and BLAST ordering by $2 \mathrm{~dB}$ at an outage probability of $10^{-3}$.

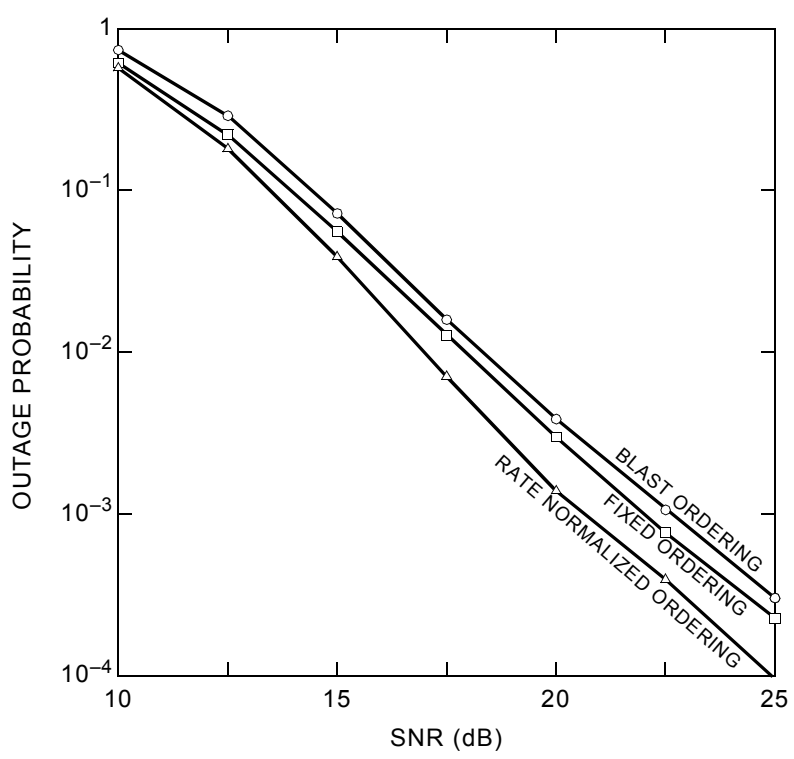

Fig. 1. Comparison of ordering algorithms for $\left\{R_{i}\right\}=\{0.00,1.31$, $2.98,3.69\}$ for a 4 -input, 4 -output Rayleigh fading channel at $R=8 \mathrm{~b} / \mathrm{s} / \mathrm{Hz}$.
Fig. 2 shows the advantage of optimizing the rate and energy allocations at the transmitter. The lowest curve shows the error performance with rate-normalized ordering and optimized transmitter allocations, while the next-lowest curve shows the performance with a fixed ordering and optimized allocations. The rate allocations for both cases are calculated anew at each SNR so as to minimize outage probability. Hence, these curves represent the best possible outage probability that can be achieved. For example, the partially uniform allocation with $K=3$ is optimal for rate-normalized ordering at $S=15 \mathrm{~dB}$, while the partially uniform allocation with $K=2$ is optimal for RN ordering at $S=20 \mathrm{~dB}$ and $25 \mathrm{~dB}$.

Comparing the lower two curves in Fig. 2, we see that transmitter-optimized rate-normalized ordering outperforms transmitter-optimized fixed ordered receiver by $1.5 \mathrm{~dB}$ at an outage probability of $10^{-3}$, underlining the importance of joint optimization of the transmitter and receiver.

Also included in Fig. 2, for the sake of comparison, is the upper gray curve, which shows the performance of a conventional BLAST system with uniform allocations at the transmitter. It is seen that the jointly optimal system outperforms conventional BLAST by $15 \mathrm{~dB}$ at an outage probability of $10^{-3}$.

\section{CONCLUSIONS}

We studied the BLAST system with successive cancellation decoding with the objective of minimizing the outage probability. We proposed the rate-normalized ordered detector and proved that it minimizes the outage probability. We investigated the optimal rate and energy allocations to minimize the outage probability for the different variants of the SC decoder. We argued that the partially uniform rate and

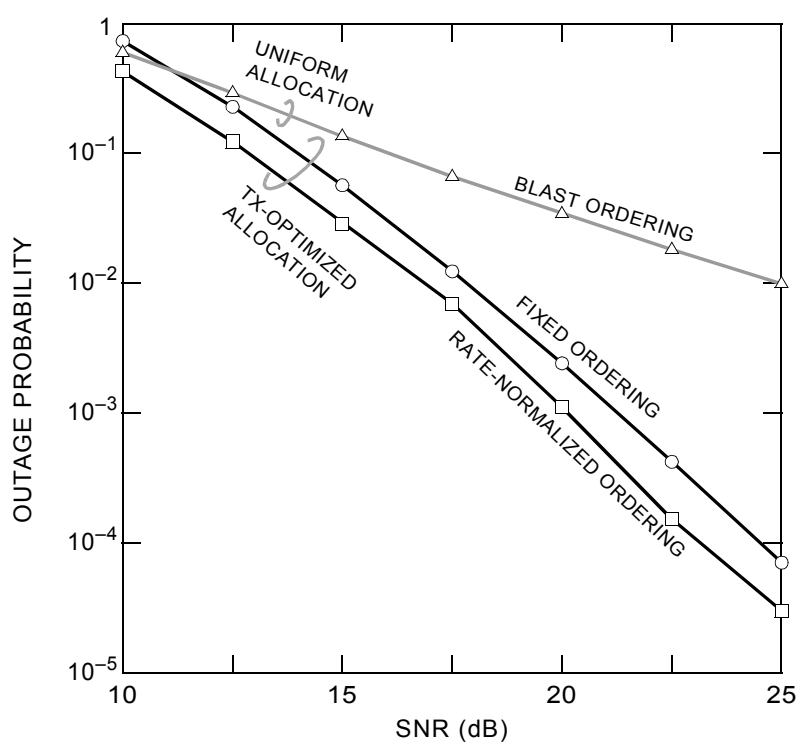

Fig. 2. Comparison of transmitter optimized SC decoders for a 4input, 4-output Rayleigh fading channel at $R=8 \mathrm{~b} / \mathrm{s} / \mathrm{Hz}$. 
energy (PURE) allocation is the minimizing solution for BLAST-ordered detection. In the case of the rate-normalized ordered decoder, we proposed a rule for rate and power allocation to minimize the outage probability based on partial analytical results. Simulation results show that jointly optimizing the transmitter and receiver, without any form of channel feedback, improves the performance of SC decoders significantly. The fact that a PURE allocation is often the optimum solution with $\mathrm{RN}$ ordering validates the importance of antenna selection schemes with SC decoding. Intuitively, antenna selection with SC decoding amounts to reducing the multiplexing gain of the MIMO system in order to improve the diversity order. Our results show the importance of achieving the right trade-off in order to minimize the outage probability of the SC decoder.

\section{Appendix 1: Proof of Theorem 1}

The BLAST ordering algorithm can be viewed as a function $\Psi(\mathbf{H})$ of the channel matrix $\mathbf{H}$, which outputs the pair $(\boldsymbol{i}, \Gamma)$.

Lemma 6.1. For a given channel matrix $\mathbf{H}$, suppose $\Psi(\mathbf{H})$ $=(\boldsymbol{i}, \Gamma)$. Then, for all column permutation matrices, $\Pi$,

$$
\Psi(\mathbf{H} \Pi)=\left(\Pi^{\mathrm{T}} \boldsymbol{i}, \Gamma\right) .
$$

Proof: Suppose the symbol $x_{q}$ corresponding to $i_{1}=q$ was decoded in the first stage with channel $\mathbf{H}$, the same symbol, re-labelled as $i_{1}{ }^{\prime}=q^{\prime}$, where $\boldsymbol{i}^{\prime}=\Pi^{T} \boldsymbol{i}$ will be decoded in the first stage with the permuted channel НП. Clearly, the value of the maximum SNR scaling factor remains unchanged for that stage, since it corresponds to the same symbol. Similarly, proceeding through the stages $k=\{2,3, \ldots, t\}$, it is clear that the SNR scaling factors remain invariant to the permutation, and that multiplying $\mathbf{H}$ by $\Pi$ amounts to re-labelling the index of the symbols, as determined by $\Pi$.

It is well known that permuting the columns of Rayleigh fading matrices does not change their distribution. More precisely, the following result holds.

Lemma 6.2. Suppose $\mathbf{H}$ is a Rayleigh fading matrix. Then, for all column permutation matrices $\Pi$, the random matrix $\mathbf{H}^{\prime}=\mathbf{H} \Pi$ is identical in distribution to $\mathbf{H}$, since $\mathbf{H}$ is circularly symmetric.

From Lemma 6.2, $\mathbf{H}$ is identical in distribution to $\mathbf{H}$. Using Lemma 6.1, we arrive at the following corollary.

Corollary 1. $\Psi(\mathbf{H} \Pi)=\left(\Pi^{T} \boldsymbol{i}, \Gamma\right)$ is identical in distribution to $\Psi(\mathbf{H})=(\boldsymbol{i}, \Gamma)$.

From Corollary 1 , since $\Pi^{T} \boldsymbol{i}$ is identical in distribution to $\boldsymbol{i}$, we conclude that $\boldsymbol{i}$ is uniformly distributed over all permutations of $[1,2, \ldots, t]^{T}$, as stated in Theorem 1 .
Further, from Corollary 1, note that joint density function of $(\boldsymbol{i}, \Gamma)$ satisfies $p(\boldsymbol{i}, \Gamma)=p\left(\Pi^{T} \boldsymbol{i}, \Gamma\right)$. Now, using Bayes' rule and the fact $\boldsymbol{i}$ is uniformly distributed over $t$ ! possibilities, we obtain the following expression for the joint density function.

$$
p(\boldsymbol{i}, \Gamma)=\frac{1}{t !} p(\Gamma \mid \boldsymbol{i})
$$

In particular, $p(\boldsymbol{i}, \Gamma)=p\left(\Pi^{T} \boldsymbol{i}, \Gamma\right) \Leftrightarrow p(\Gamma \mid \boldsymbol{i})=p\left(\Gamma \mid \Pi^{T} \boldsymbol{i}\right)$ for all $\Pi$, implying that $\Gamma$ and $\boldsymbol{i}$ are independent. This proves the second claim of Theorem 1.

\section{REFERENCES}

[1] P. W. Wolniansky, G. J. Foschini, G. D. Golden, R. A. Valenzuela, VBLAST: An Architecture for Realizing Very High Data Rates Over the Rich-Scattering Wireless Channel, Proc. ISSSE-98, Pisa, Italy, Sept. 29, 1998.

[2] G. D. Golden, G. J. Foschini, R. A. Valenzuela, P. W. Wolniansky, Detection Algorithm and Initial Laboratory Results using the VBLAST Space-Time Communication Architecture, Electronics Letters, Vol. 35, No. 1, Jan. 7, 1999, pp. 14-15.

[3] S. T. Chung, A. Lozano and H. C. Huang, "Approaching eigenmode BLAST channel capacity using V-BLAST with rate and power feedback", in Proc. VTC 2001, Atlantic City, Oct. 2001.

[4] S. H. Nam and K. B. Lee, "Transmit power allocation for an extended V-BLAST system”, Proc. IEEE PIMRC 2002, Sept. 2002, pp. 843848.

[5] R. W. Heath Jr., S. Sandhu, and A. J. Paulraj, "Antenna Selection for spatial multiplexing systems with linear receivers", IEEE Communication Letters, vol. 5, no. 4, pp 142-144, April 2001.

[6] N. Prasad and M. K. Varanasi, "Outage Analysis and Optimization for Multiaccess/V-BLAST Architecture over MIMO Rayleigh Fading Channels", 41st Annual Allerton Conf. on Comm. Control, and Comput., Monticello, IL, Oct., 2003.

[7] T. Guess, H. Zhang, and T. V. Kotchiev, "The outage capacity of BLAST for MIMO channels," in Proc. IEEE Intl. Conf. on Communications, Anchorage, Alaska, May 2003.

[8] H. Zhang and T. Guess, "Asymptotical analysis of the outage capacity of rate-tailored BLAST", Proc. IEEE Globecom 2003. Vol. 4, Dec. 2003 pp. 1797-1801.

[9] Ravi Narasimhan, "Spatial Multiplexing with transmit antenna and constellation selection for correlated MIMO fading channels", IEEE Transactions on Signal Processing, Special Issue on MIMO Wireless Communications, vol. 51, no. 11, pp 2829-2838, Nov. 2003

[10] D. A. Gore, R. W. Heath Jr. and A. J. Paulraj, "Statistical antenna selection for spatial multiplexing systems", IEEE Int. Conf. On Communications, vol 1. pp 450-454, 2002.

[11] G. D. Forney Jr. and M. V. Eyuboglu, "Combined Equalization and Coding using Precoding", IEEE Communications Magazine, Dec. 1991.

[12] R. J. Muirhead, Aspects of Multivariate Statistical Theory, John Wiley \& Sons, 1982.

[13] J. R. Barry, E. A. Lee, D. G. Messerschmitt, Digital Communication, Third Edition, Kluwer Academic Publishers, 2004. 